OPEN ACCESS

Edited by:

Mehdi Razzaghi-Abyaneh,

Pasteur Institute of Iran, Iran

Reviewed by:

Soner Soylu,

Mustafa Kemal University, Turkey

Masoomeh Shams-Ghahfarokhi,

Tarbiat Modares University, Iran

Loredana Scalschi,

Tel Aviv University, Israel

*Correspondence:

Xingfeng Shao

shaoxingfeng@nbu.edu.cn

Specialty section:

This article was submitted to

Food Microbiology,

a section of the journal

Frontiers in Microbiology

Received: 10 April 2017

Accepted: 22 May 2017

Published: 06 June 2017

Citation:

Xu J, Shao X, Li Y, Wei Y, XU F and Wang $H$ (2017) Metabolomic Analysis and Mode of Action of Metabolites of

Tea Tree Oil Involved in the

Suppression of Botrytis cinerea.

Front. Microbiol. 8:1017.

doi: 10.3389/fmicb.2017.01017

\section{Metabolomic Analysis and Mode of Action of Metabolites of Tea Tree Oil Involved in the Suppression of Botrytis cinerea}

\author{
Jiayu Xu, Xingfeng Shao *, Yonghua Li, Yingying Wei, Feng Xu and Hongfei Wang \\ Department of Food Science and Engineering, Ningbo University, Ningbo, China
}

Tea tree oil (TTO), a volatile essential oil, has been widely used as an antimicrobial agent. However, the mechanism underlying TTO antifungal activity is not fully understood. In this study, a comprehensive metabolomics survey was undertaken to identify changes in metabolite production in Botrytis cinerea cells treated with $\Pi \mathrm{T}$. Significant differences in 91 metabolites were observed, including 8 upregulated and 83 downregulated metabolites in TTO-treated cells. The results indicate that TTO inhibits primary metabolic pathways through the suppression of the tricarboxylic acid (TCA) cycle and fatty acid metabolism. Further experiments show that TTO treatment decreases the activities of key enzymes in the TCA cycle and increases the level of hydrogen peroxide $\left(\mathrm{H}_{2} \mathrm{O}_{2}\right)$. Membrane damage is also induced by TTO treatment. We hypothesize that the effect of TTO on B. cinerea is achieved mainly by disruption of the TCA cycle and fatty acid metabolism, resulting in mitochondrial dysfunction and oxidative stress.

Keywords: tea tree oil, Botrytis cinerea, antifungal mechanism, metabolomics, TCA cycle

\section{INTRODUCTION}

Botrytis cinerea is a necrotrophic fungal pathogen that causes gray mold, a seriously destructive postharvest disease that affects a variety of fresh fruits (Saito et al., 2016). Even at low temperature during storage and transportation, it can produce significant degradation in fruit quality, resulting in severe economic losses. Because chemical fungicides are harmful to human health and the environment, there is an urgent need to develop safe and effective antifungal agents to control the decay caused by $B$. cinerea. Natural antimicrobial agents such as essential oils provide one promising alternative. For example, the essential oils of Origanum compactum and Thymus glandulosus strongly inhibit the growth of mycelia in B. cinerea (Bouchra et al., 2003), and essential oils from three Monarda species have antifungal effects on both mycelia and spores (Adebayo et al., 2013). Syzygium aromaticum, Brassica nigra, and Solidago canadensis L. essential oils also exhibit effective antifungal activity in strawberries against $B$. cinerea in vitro and in vivo (Aguilar-González et al., 2015; Liu et al., 2016).

Tea tree oil (TTO), the volatile essential oil extracted from leaves and branches of the Australian native plant Melaleuca alternifolia by steam distillation, is frequently used as a topical medicinal and 
antimicrobial agent (Pazyar et al., 2013). Because of its bioactivities, its efficacy has recently been evaluated against Staphylococcus aureus, Escherichia coli, Aspergillus niger, and Candida albicans in vitro (Gustafson et al., 1998; Hammer et al., 2003; Shi et al., 2016). TTO controls the growth of $B$. cinerea more effectively than pine and cinnamon oil (Szczerbanik et al., 2007), or clove oil and garlic oil (Cheng and Shao, 2011). TTO also effectively inhibits the growth of $B$. cinerea on Dutch White cabbage (Bishop and Reagan, 1998) and exhibits high antifungal activity on B. cinerea in strawberry fruit (Shao et al., 2013b).

Essential oils are generally thought to exert their antifungal activity by damaging plasma membranes (Hood et al., 2010; Khan et al., 2010) and mitochondria (Tian et al., 2012; Zheng et al., 2015; Li et al., 2016). Microscopic observations revealed that the essential oils of Origanum syriacum L. var. bevanii and Lavandula stoechas L. var. stoechas caused considerable morphological degenerations of the fungal hyphae of $B$. cinerea such as cytoplasmic coagulation, vacuolations, hyphal shriveling and protoplast leakage, and loss of conidiation (Soylu et al., 2010). Our previous studies confirmed that TTO primarily targets the $B$. cinerea cell wall, resulting in increased membrane permeability, the release of cellular material, and eventual cell death (Shao et al., 2013a). Terpinen-4-ol, the major characteristic component of TTO, severely compromises membrane integrity and increases permeability. Another characteristic component in TTO, 1,8-cineole, damages cellular organelles but does not affect membrane permeability (Yu et al., 2015). These findings are important clues for understanding TTO antifungal activity, although the underlying mechanisms are still not fully clear.

Metabolomics is an emerging technology that provides a comprehensive quantitative and qualitative inventory of the low molecular weight metabolites in a cell or organism (Fiehn et al., 2000). It is particularly useful for analyzing changes in the endogenous metabolism of a biological system that has been stimulated or disturbed, and for identifying metabolic pathways. Recently, metabolomics has been widely applied to study the mechanisms by which different agents exert their antimicrobial effects against fungi and bacteria. For example, the activity of amphotericin B against C. albicans can be attributed to changes in metabolite production (Cao et al., 2013). Another study found that the microbiostatic effect of $\varepsilon$-Poly-L-lysine on Saccharomyces cerevisiae is achieved by breaking the balance of intracellular metabolites due to disruptions in cell membrane function (Bo et al., 2014). Finally, a metabolomics approach was used to demonstrate that cinnamaldehyde changes the metabolism of $E$. coli by interacting with different biochemical targets (Mousavi et al., 2016).

No studies have yet employed metabolomics to examine changes in fungi in the presence of essential oils. In order to examine the antifungal effects of TTO on $B$. cinerea in detail, we conducted a metabolomics analysis using an ultra-high pressure liquid chromatography system coupled to a quadrupole time-offlight mass spectrometer (UHPLC-Q-TOF MS).

This study was designed to reveal the mechanisms responsible for the antifungal effects and stress caused by TTO in B. cinerea by generating a high-resolution metabolic fingerprint and detecting changes in metabolite levels at high sensitivity.

\section{MATERIALS AND METHODS}

\section{Fungal Isolates, Essential Oil, and Reagents}

A highly virulent strain of $B$. cinerea was isolated from strawberries and cultured at $25^{\circ} \mathrm{C}$ on potato dextrose agar medium (PDA; containing $1 \mathrm{~L}$ of an infusion from potatoes, 20 $\mathrm{g} / \mathrm{L}$ glucose, and $15 \mathrm{~g} / \mathrm{L}$ agar) before use in experiments. TTO was purchased from Fuzhou Merlot Lotus Biological Technology Company (Fujian Province, China). Ammonium acetate and ammonium hydroxide were purchased from Sigma Aldrich (St. Louis, MO, USA). Acetonitrile and methanol were purchased from Merck (Germany). Ammonium acetate and acetonitrile were of HPLC-grade. Distilled water was filtered through a Milli-Q system from EMD Millipore Corporation (Billerica, MA, USA). Succinate dehydrogenase (SDH), malic dehydrogenase $(\mathrm{MDH})$, citrate synthase (CS), isocitrate dehydrogenase (ICDH), $\alpha$-ketoglutarate dehydrogenase $(\alpha-\mathrm{KGDH})$, and hydrogen peroxide $\left(\mathrm{H}_{2} \mathrm{O}_{2}\right)$ reagent kits were purchased from Nanjing Jian Cheng Bioengineering Institute (Nanjing, China).

\section{Fungal Culture and Sample Preparation}

$B$. cinerea cultures were maintained on PDA at $25^{\circ} \mathrm{C}$ for 3 days. The spore suspension was harvested by adding $10 \mathrm{~mL}$ sterile $0.9 \% \mathrm{NaCl}$ solution to each petri dish and gently scraping the mycelial surface three times with a sterile L-shaped spreader to free the spores. The spore suspension was adjusted using a hemocytometer to $5 \times 10^{6}$ spores $/ \mathrm{mL}$. One milliliter suspension was inoculated into $250 \mathrm{~mL}$ flasks containing $150 \mathrm{~mL}$ sterile potato dextrose broth (PDB; containing $1 \mathrm{~L}$ of an infusion from potatoes, $20 \mathrm{~g} / \mathrm{L}$ glucose) medium and cultured at $25^{\circ} \mathrm{C}$ on a rotary shaker at 150 revolutions per minute (rpm) for 3 days. TTO was added to the medium to a final concentration of $5 \mathrm{~mL} / \mathrm{L}$, and cultures incubated for $2 \mathrm{~h}$. Mycelia were then collected, rinsed three times with $0.1 \mathrm{M}$ phosphate buffer saline (PBS; $\mathrm{pH}=7.4$ ), and immediately frozen in liquid nitrogen. Samples were stored at $-80^{\circ} \mathrm{C}$. Cultures without TTO were used as a control. Six samples of each group were prepared in parallel for LC-MS/MS analysis and other tests.

\section{LC-MS/MS Analysis}

Before analysis, control and treated mycelia $(\sim 100 \mathrm{mg}$ wet weight) were thawed at $4{ }^{\circ} \mathrm{C}$ and homogenized. One milliliter of acetonitrile and methanol $(1: 1, \mathrm{v} / \mathrm{v})$ was added to each sample and mixed by vortexing. Samples were immediately frozen in liquid nitrogen, subjected to ultrasonic grinding for $10 \mathrm{~min}$, stirred for $60 \mathrm{~min}$ at $-20^{\circ} \mathrm{C}$, and then separated by centrifugation at 12,000 $\times \mathrm{g}$ for $15 \mathrm{~min}$ at $4^{\circ} \mathrm{C}$. An aliquot of $900 \mu \mathrm{L}$ supernatant was dried under vacuum, dissolved in $100 \mu \mathrm{L}$ of acetonitrile and water $(1: 1, \mathrm{v} / \mathrm{v})$ by vortexing, and centrifuged at $12,000 \times \mathrm{g}$ for $15 \mathrm{~min}$ at $4^{\circ} \mathrm{C}$. The chemical compositions of the samples were analyzed using an UHPLC (1290 Infinity LC, Agilent Technologies) equipped with a quadrupole time-of-flight (AB Sciex TripleTOF 6600) mass spectrometer.

For separation by hydrophilic interaction liquid chromatography (HILIC), samples were analyzed using a $2.1 \mathrm{~mm} \times 100 \mathrm{~mm}$ ACQUIY UPLC BEH $1.7 \mu \mathrm{m}$ column 
(Waters Corporation, Ireland). In both electron spray ionization (ESI) positive and negative modes, the mobile phase contained (A) $25 \mathrm{mM}$ ammonium acetate and $25 \mathrm{mM}$ ammonium hydroxide in water, and (B) acetonitrile. The elution gradient was: $0-1 \mathrm{~min}, 85 \% \mathrm{~B}$; $1-12 \mathrm{~min}, 85-65 \% \mathrm{~B} ; 12-12.1 \mathrm{~min}, 65-40 \%$ B; $12.1-15 \mathrm{~min}, 40 \% \mathrm{~B} ; 15-15.1 \mathrm{~min}, 40-85 \% \mathrm{~B} ; 15.1-20 \mathrm{~min}$, $85 \% \mathrm{~B}$, with a $5 \mathrm{~min}$ re-equilibration period employed.

The ESI source conditions were set as follows: Ion Source Gas1 (Gas1) as 60, Ion Source Gas2 (Gas2) as 60, curtain gas (CUR) as 30, source temperature: $600^{\circ} \mathrm{C}$, Ion Spray Voltage Floating (ISVF) $\pm 5,500 \mathrm{~V}$. In the MS only acquisition mode, the instrument was set to acquire over the $\mathrm{m} / \mathrm{z}$ range $60-$ $1,000 \mathrm{Da}$, and the accumulation time for the TOF MS scan was set at $0.20 \mathrm{~s} /$ spectrum. In the auto MS/MS acquisition mode, the instrument was set to acquire over the $\mathrm{m} / \mathrm{z}$ range 25-1,000 Da, and the accumulation time for product ion scan was set at $0.05 \mathrm{~s} /$ spectrum. The product ion scan was acquired using information dependent acquisition (IDA) with the high sensitivity mode selected. The collision energy (CE) was fixed at $35 \mathrm{~V} \pm 15 \mathrm{eV}$. The declustering potential (DP) was set to $\pm 60 \mathrm{~V}$.

\section{Determination of Effect of TTO on the Leakage of Cell Membrane}

In order to assess TTO affect on the leakage of cell membrane, the absorbance at $260 \mathrm{~nm}$ of $B$. cinerea cell was determined. As described above for fungal culture and sample preparation, TTO was added to PDB medium at a final concentration of $5 \mathrm{~mL} / \mathrm{L}$. PDB without TTO served as the control. Treated and control group were centrifuged at $10,000 \times g$ for $10 \mathrm{~min}$ at $4^{\circ} \mathrm{C}$, and the absorbance of the supernatant was measured immediately at $260 \mathrm{~nm}$ with a UV/Vis spectrophotometer (UV-2000, UNICO Instrument Co., Ltd., Shanghai, China). After $2 \mathrm{~h}$ incubation with or without TTO, treated and control group were centrifuged and the absorbance at $260 \mathrm{~nm}$ of the obtained supernatant were measured. Each treatment was performed in triplicates. The percentage change in $\mathrm{A}_{260 \mathrm{~nm}}$ (Liu et al., 2004) was calculated using the following formula:

$$
\mathrm{A}_{260 \mathrm{~nm}} \text { percentage change }(\%)=\left[\left(\mathrm{A}_{\mathrm{t}}-\mathrm{A}_{0}\right) / \mathrm{A}_{0}\right] \times 100
$$

where $A_{0}$ is the mean $A_{260} \mathrm{~nm}$ for cultures measured after $0 \mathrm{~h}$ of treatment, and $A_{t}$ is the mean $A_{260 n m}$ for cultures after $2 \mathrm{~h}$ of treatment.

\section{Activities of Key Enzymes Involved in Tricarboxylic Acid (TCA) Cycle}

Control and treated mycelia were washed with $0.1 \mathrm{M} \mathrm{PBS}(\mathrm{pH}=$ 7.4) three times and then ground in liquid nitrogen. The ground material was suspended in $10 \mathrm{~mL} 0.05 \mathrm{M}$ PBS $(\mathrm{pH}=7.2)$ and centrifuged at $10,000 \times g$ for $10 \mathrm{~min}$ at $4^{\circ} \mathrm{C}$. Enzyme activities were measured in the supernatant for $\mathrm{SDH}, \mathrm{MDH}, \mathrm{CS}, \mathrm{ICDH}$, and $\alpha-\mathrm{KGDH}$, using commercially available kits purchased from Nanjing Jiancheng Bioengineering Institute (Nanjing, China) following the manufacturer's instructions. ICDH, $\mathrm{MDH}$, and $\alpha-\mathrm{KGDH}$ activities were detected at $340 \mathrm{~nm}$ in redox reaction assays. SDH and CS activities were determined at 600 and $412 \mathrm{~nm}$, respectively. All tests were performed in triplicate.

\section{Determination of Effect of TTO on $\mathrm{H}_{2} \mathrm{O}_{2}$ Accumulation}

Control and treated mycelia ( $1 \mathrm{~g}$ wet weight) were mixed with $5 \mathrm{~mL} 0.05$ M PBS ( $\mathrm{pH}=7.0$, containing $3 \%$ polyvinyl pyrrolidone), subjected to ultrasonic grinding for $15 \mathrm{~min}$, and centrifuged at $10,000 \times g$ for $10 \mathrm{~min}$ at $4^{\circ} \mathrm{C}$. The supernatant was used to determine $\mathrm{H}_{2} \mathrm{O}_{2}$ content of the fungal cells using assay kits obtained from Nanjing Jiancheng Institute of Bioengineering (Nanjing, Jiangsu, China) following the manufacturer's instructions. $\mathrm{H}_{2} \mathrm{O}_{2}$ content was expressed as $\mathrm{mmol} / \mathrm{g}$ prot. All tests were performed in triplicate.

\section{Data Processing}

The raw MS data (.wiff scan files) were converted to MzXML format using ProteoWizard MSConvert and processed using XCMS for feature detection, retention time correction, and alignment. Metabolites were identified by matching high accuracy $(<25 \mathrm{ppm})$ MS/MS data to our standards database.

\section{Statistical Analysis}

In the extracted ion features, only variables having more than $50 \%$ of the non-zero measurement values in at least one group were retained. For multivariate statistical analysis, the Metabo Analyst (http://www.metaboanalyst.ca) web-based system was used. After Pareto scaling, principal component analysis (PCA) and partial least-squares-discriminant analysis (PLS-DA) were performed. Leave-one-out cross-validation and response permutation testing were used to evaluate the robustness of the model. Metabolites exhibiting significant differences were identified based on the combination of a statistically significant threshold of variable influence on projection (VIP) values obtained from a PLS-DA model and a two-tailed Student's $t$-test ( $p$-value) on the raw data. Metabolites with VIP-values larger than 1.0 and $p<0.05$ were considered significant. All enzyme activity and $\mathrm{H}_{2} \mathrm{O}_{2}$ content data were analyzed using SAS software (Version 8.2; SAS Institute, Cary, NC, USA). These data were analyzed by one-way analysis of variance (ANOVA). Comparison of means was performed by Duncan's multiple range tests. A value of $P<0.05$ was considered statistically significant.

\section{RESULTS}

\section{Multivariate Analysis}

Extracts from TTO-treated and untreated $B$. cinerea were analyzed using a UHPLC-Q-TOF mass spectrometer in positive and negative ion modes. Multivariate analysis was applied to find potential biomarkers. PCA, an unsupervised pattern recognition method, was performed to examine intrinsic variation in the data set. Samples that have similar metabolomics profiles are clustered together and those that are different are placed further apart in PCA score plots. As shown by the PCA score plots in positive (Figure 1A) and negative (Figure 1B) ion modes, the control group and TTO-treated group clustered separately, which indicates that metabolite levels were altered in the TTO-treated group.

To further identify ion peaks that could discriminate between the groups, a supervised pattern recognition method (PLS-DA) 


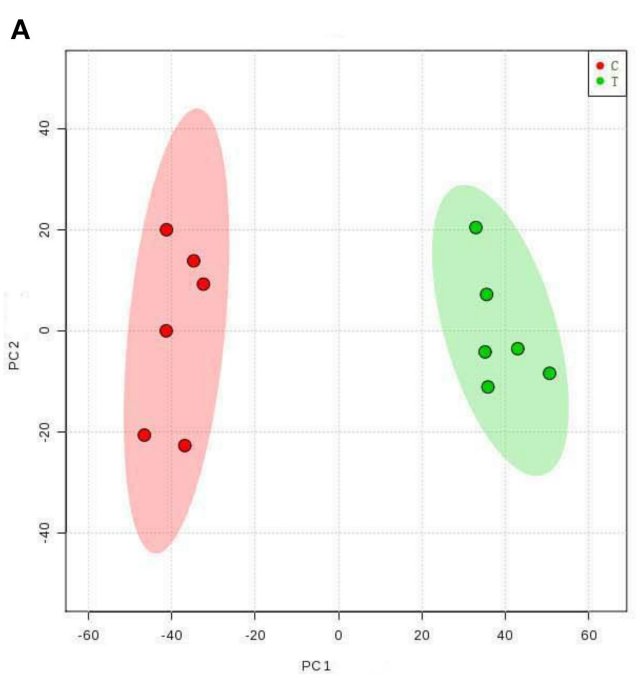

C

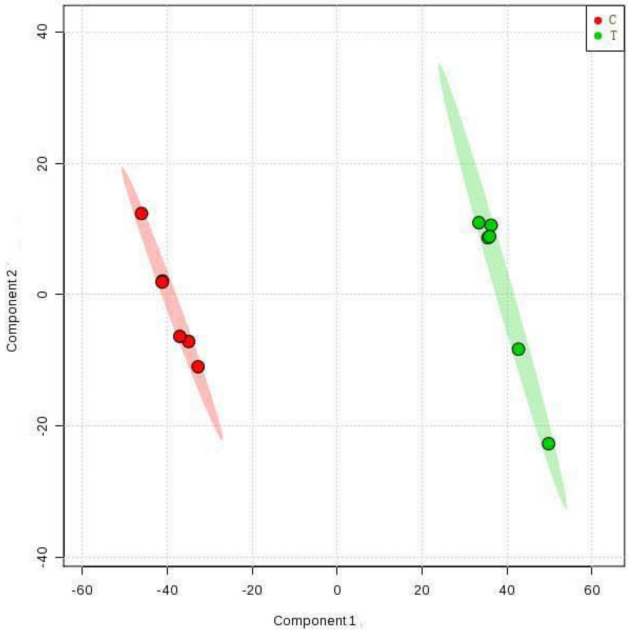

B

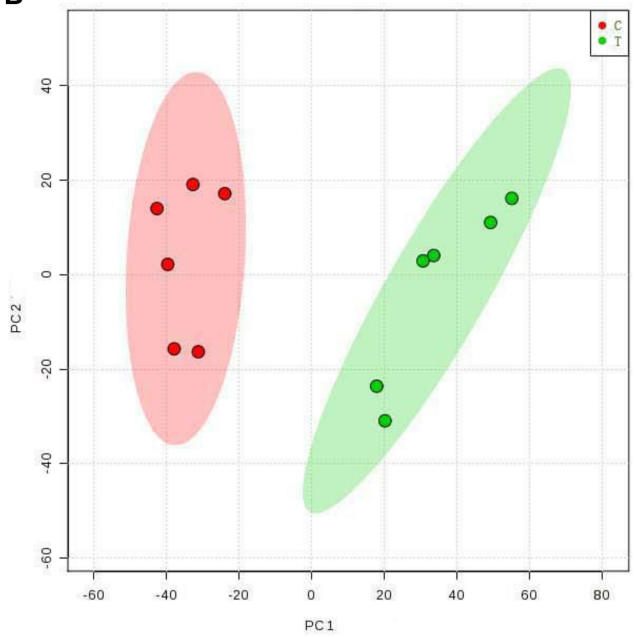

D

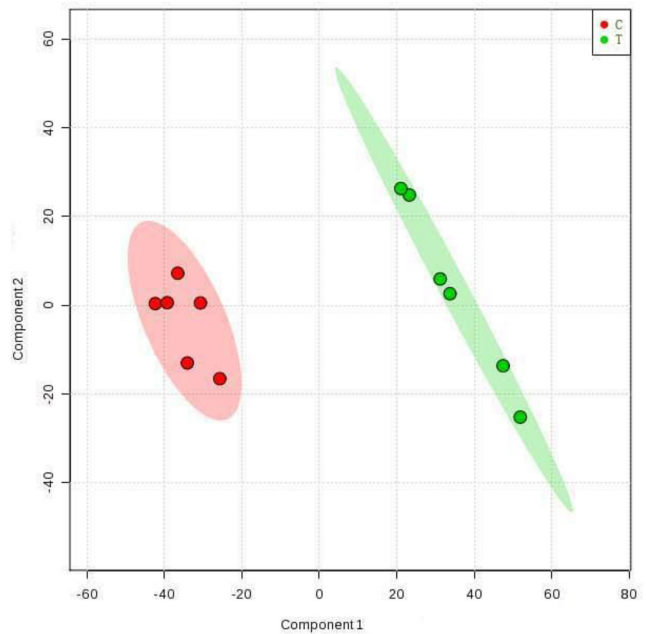

FIGURE 1 | Score plots representing PCA and PLS-DA results based on the UHPLC-Q-TOF MS spectra. B. cinerea mycelia from the TTO-treated cells (green dots) and control cells (red dots) are shown in (A,C) positive and (B,D) negative modes. C, control sample; T, TTO-treated sample.

was suitable for the selection of biomarkers in similar metabolic profiling data (Schneider et al., 2009). In the PLS-DA score plots for both the positive (Figure 1C) and negative (Figure 1D) ion modes, metabolic differences made it possible to resolve the control and TTO-treated groups into distinct clusters. The $R^{2}$ and $Q^{2}$ parameters are used to evaluate the quality of the PLSDA model. The $R^{2}$ and $Q^{2}$ were 0.99 and 0.97 in positive ion mode, respectively, and $R^{2}$ and $Q^{2}$ were 0.99 and 0.96 in negative ion mode, indicating that the model had high predictability and reliability.

\section{Selection and Identification of Biomarkers}

To find the endogenous metabolites that contributed most to the separation of the control and TTO-treated samples, VIP scores were determined after PLS-DA. VIP measures the contribution made by each variable to the PLS-DA model. Ions were considered as potential biomarkers if they satisfied the criteria
VIP-value $>1$ and $p$-value $<0.05$. Based on this algorithm, 91 metabolite ions were identified as primary contributors to the separation between control and TTO-treated groups. The data are summarized in Table 1, which also classifies the metabolites as either upregulated ( 8 metabolites) or downregulated (83 metabolites).

\section{Hierarchical Clustering Analysis}

Table 1 shows the metabolic changes in fungal cells after treatment with TTO. The metabolites identified include products and intermediates generated by the TCA cycle, fatty acids, amino acids, and carbohydrates. The results suggest that $B$. cinerea adapts to environmental changes by regulating metabolic pathways. To provide an intuitive representation of the discriminatory power of the selected biomarkers in untreated and TTO-treated B. cinerea, a visual hierarchical clustering analysis (HCA, Figure 2) was performed. Each 
TABLE 1 | Identification and changes in levels of potential biomarkers in B. cinerea cells.

\begin{tabular}{|c|c|c|c|c|c|c|c|c|}
\hline Metabolite & Ionization mode & RT (min) & VIP & $\begin{array}{c}\text { Fold } \\
\text { change }\end{array}$ & $P$-value & $\mathrm{m} / \mathbf{z}$ & $\begin{array}{l}\text { Trend (treated } \\
\text { vs. control) }\end{array}$ & Functional category \\
\hline Trehalose & $\mathrm{ESI}(-)$ & 8.68 & 1.75 & 0.08 & $8.53 \times 10^{-7}$ & 401.12816 & $\downarrow$ & Oxidative stress \\
\hline Myristic acid & $\mathrm{ESI}(-)$ & 1.18 & 1.87 & 0.06 & $3.89 \times 10^{-5}$ & 287.22120 & $\downarrow$ & Lipid metabolism \\
\hline L-Malic acid & ESI(-) & 11.17 & 1.41 & 0.17 & $2.02 \times 10^{-5}$ & 133.01430 & $\downarrow$ & TCA cycle \\
\hline L-Glutamate & ESI(+), ESI(-) & 8.51 & 1.52 & 0.13 & $1.41 \times 10^{-7}$ & 146.04635 & $\downarrow$ & Amino acid metabolism \\
\hline Isocitrate & $\mathrm{ESI}(-)$ & 11.22 & 1.24 & 0.20 & $2.12 \times 10^{-3}$ & 191.01933 & $\downarrow$ & TCA cycle \\
\hline Hypoxanthine & ESI(+), ESI(-) & 2.37 & 1.20 & 0.24 & $3.44 \times 10^{-4}$ & 135.03135 & $\downarrow$ & Purine metabolism \\
\hline Xanthine & $\mathrm{ESI}(+)$ & 3.44 & 1.13 & 0.32 & $1.10 \times 10^{-4}$ & 153.03962 & $\downarrow$ & Purine metabolism \\
\hline $\mathrm{PC}(16: 0 / 16: 0)$ & $\mathrm{ESI}(+)$ & 1.00 & 1.13 & 0.32 & $7.10 \times 10^{-5}$ & 778.53738 & $\downarrow$ & Lipid metabolism \\
\hline L-Proline & $\mathrm{ESI}(+)$ & 1.73 & 1.94 & 0.05 & $2.21 \times 10^{-3}$ & 157.09589 & $\downarrow$ & Amino acid metabolism \\
\hline L-Carnosine & $\mathrm{ESI}(+)$ & 6.52 & 1.88 & 8.54 & $4.06 \times 10^{-5}$ & 227.11334 & $\uparrow$ & Amino acid metabolism \\
\hline L-Arginine & $\mathrm{ESI}(+)$ & 14.07 & 1.06 & 0.26 & 0.05 & 175.11853 & $\downarrow$ & Amino acid metabolism \\
\hline Imidazoleacetic acid & $\mathrm{ESI}(+)$ & 7.40 & 1.47 & 3.44 & $1.61 \times 10^{-6}$ & 270.11914 & $\uparrow$ & Amino acid metabolism \\
\hline $\mathrm{GMP}^{(1)}$ & $\mathrm{ESI}(+)$ & 10.42 & 1.04 & 0.37 & $1.09 \times 10^{-5}$ & 364.06465 & $\downarrow$ & Purine metabolism \\
\hline Guanosine & $\mathrm{ESI}(+), \mathrm{ESI}(-)$ & 4.04 & 1.17 & 2.27 & 0.04 & 282.08322 & $\uparrow$ & Purine metabolism \\
\hline D-Mannitol & $\mathrm{ESI}(+)$ & 5.49 & 2.00 & 0.06 & $2.54 \times 10^{-8}$ & 183.08529 & $\downarrow$ & $\begin{array}{l}\text { Fructose and mannose } \\
\text { metabolism }\end{array}$ \\
\hline $\begin{array}{l}\text { D-Glucose } \\
\text { 6-phosphate }\end{array}$ & $\mathrm{ESI}(+), \mathrm{ESI}(-)$ & 11.68 & 1.55 & 0.16 & $1.50 \times 10^{-6}$ & 278.06307 & $\downarrow$ & Glycolysis/gluconeogenesis \\
\hline D-Glucose & $\mathrm{ESI}(+)$ & 11.68 & 1.57 & 0.15 & $4.24 \times 10^{-5}$ & 243.02608 & $\downarrow$ & Glycolysis/gluconeogenesis \\
\hline UDP & $\mathrm{ESI}(+)$ & 10.44 & 1.96 & 0.07 & $1.75 \times 10^{-7}$ & 405.00844 & $\downarrow$ & Pyrimidine metabolism \\
\hline $\begin{array}{l}\text { UDP-N- } \\
\text { acetylglucosamine }\end{array}$ & ESI(+), ESI(-) & 10.00 & 2.18 & 0.04 & $2.07 \times 10^{-8}$ & 608.08737 & $\downarrow$ & Peptidoglycan biosynthesis \\
\hline UDP-D-Glucose & ESI(-) & 10.42 & 1.90 & 0.06 & $4.60 \times 10^{-5}$ & 565.04443 & $\downarrow$ & Galactose metabolism \\
\hline UDP-D-Galactose & $\mathrm{ESI}(+)$ & 10.44 & 2.00 & 0.06 & $2.03 \times 10^{-7}$ & 584.08782 & $\downarrow$ & Galactose metabolism \\
\hline Thiamine & $\mathrm{ESI}(+)$ & 8.07 & 1.20 & 0.28 & $4.73 \times 10^{-4}$ & 265.11128 & $\downarrow$ & Vitamin metabolism \\
\hline $\begin{array}{l}\text { S-Methyl-5'- } \\
\text { thioadenosine }\end{array}$ & $\mathrm{ESI}(+), \mathrm{ESI}(-)$ & 1.28 & 1.74 & 0.07 & $1.34 \times 10^{-6}$ & 296.08064 & $\downarrow$ & Purine metabolism \\
\hline Sebacic acid & ESI(-) & 5.92 & 1.59 & 0.10 & $3.22 \times 10^{-3}$ & 201.11226 & $\downarrow$ & Lipid metabolism \\
\hline $\mathrm{GMP}^{(2)}$ & $\mathrm{ESI}(+)$ & 10.42 & 1.04 & 0.37 & $1.09 \times 10^{-5}$ & 364.06465 & $\downarrow$ & Purine metabolism \\
\hline Sarcosine & $\mathrm{ESI}(+)$ & 6.64 & 1.23 & 0.28 & $2.73 \times 10^{-5}$ & 90.05550 & $\downarrow$ & Amino acid metabolism \\
\hline $\begin{array}{l}\text { sn-Glycerol 3- } \\
\text { phosphoethanolamine }\end{array}$ & $\mathrm{ESI}(-)$ & 8.45 & 1.73 & 0.08 & $1.99 \times 10^{-5}$ & 214.04807 & $\downarrow$ & Lipid metabolism \\
\hline Pantothenate & $\mathrm{ESI}(+)$ & 3.96 & 2.21 & 0.03 & $9.13 \times 10^{-6}$ & 220.11742 & $\downarrow$ & Vitamin metabolism \\
\hline Nicotinate & $\mathrm{ESI}(+), \mathrm{ESI}(-)$ & 2.79 & 2.27 & 0.03 & $5.47 \times 10^{-8}$ & 124.03858 & $\downarrow$ & Vitamin metabolism \\
\hline ADMA & $\mathrm{ESI}(+)$ & 13.49 & 1.21 & 0.29 & 0.02 & 203.14994 & $\downarrow$ & Amino acid metabolism \\
\hline Phenyllactic acid & $\mathrm{ESI}(-)$ & 1.40 & 2.07 & 0.03 & $2.98 \times 10^{-4}$ & 165.05476 & $\downarrow$ & Others \\
\hline N-Acetylputrescine & $\mathrm{ESI}(+)$ & 6.97 & 1.05 & 0.21 & 0.03 & 131.11737 & $\downarrow$ & Others \\
\hline
\end{tabular}


TABLE 1 | Continued

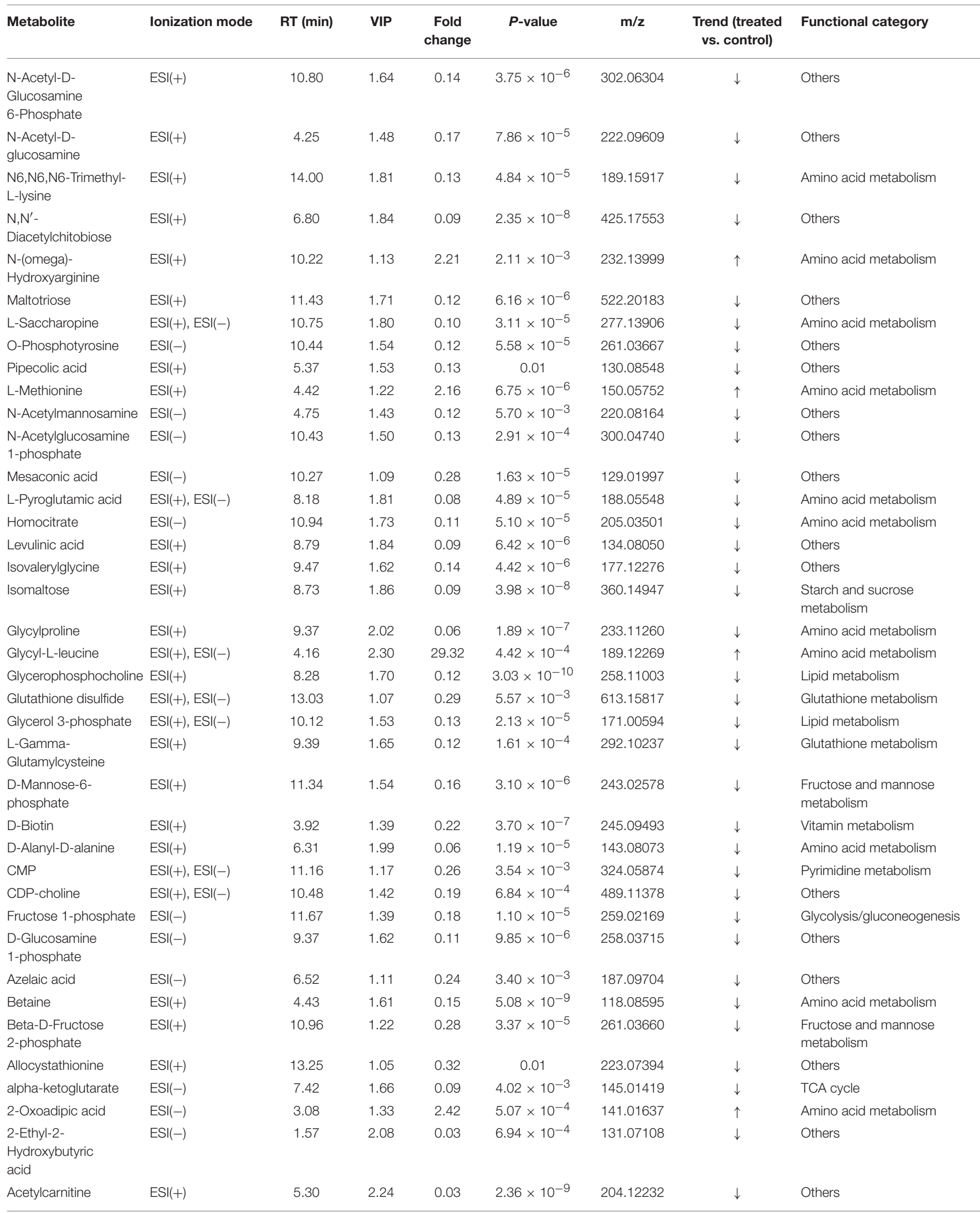


TABLE 1 | Continued

\begin{tabular}{|c|c|c|c|c|c|c|c|c|}
\hline Metabolite & Ionization mode & RT (min) & VIP & $\begin{array}{c}\text { Fold } \\
\text { change }\end{array}$ & $P$-value & $\mathrm{m} / \mathrm{z}$ & $\begin{array}{l}\text { Trend (treated } \\
\text { vs. control) }\end{array}$ & Functional category \\
\hline 7-Methylguanosine & $\mathrm{ESI}(+)$ & 5.82 & 1.29 & 0.08 & 0.10 & 298.11150 & $\downarrow$ & Purine metabolism \\
\hline 4-Pyridoxic acid & $\mathrm{ESI}(+)$ & 4.26 & 1.67 & 0.13 & $1.49 \times 10^{-7}$ & 244.07854 & $\downarrow$ & Vitamin metabolism \\
\hline $\begin{array}{l}\text { 4-Guanidinobutyric } \\
\text { acid }\end{array}$ & $\mathrm{ESI}(+)$ & 6.99 & 1.11 & 0.30 & $6.75 \times 10^{-4}$ & 146.09158 & $\downarrow$ & Others \\
\hline $\begin{array}{l}\text { 3-Hydroxyanthranilic } \\
\text { acid }\end{array}$ & $\mathrm{ESI}(+)$ & 6.58 & 1.93 & 0.07 & $2.75 \times 10^{-6}$ & 154.04900 & $\downarrow$ & Others \\
\hline 1-Methyladenosine & $\mathrm{ESI}(+)$ & 4.87 & 1.01 & 0.36 & $5.55 \times 10^{-4}$ & 282.11910 & $\downarrow$ & Purine metabolism \\
\hline (S)-2-Hydroxyglutarate & $\mathrm{ESI}(-)$ & 8.34 & 1.77 & 0.07 & $1.14 \times 10^{-3}$ & 147.02980 & $\downarrow$ & Others \\
\hline $\begin{array}{l}\text { (S)-2-aminobutyric } \\
\text { acid }\end{array}$ & ESI(-) & 8.51 & 1.55 & 0.13 & $2.87 \times 10^{-7}$ & 102.05681 & $\downarrow$ & Others \\
\hline
\end{tabular}

Trend: Relative metabolite levels in B. cinerea mycelia treated with TTO in comparison with control ( $\uparrow$, upregulated; $\downarrow$, downregulated). PC, phosphatidylcholine; AMP, adenosine monophosphate; GMP, guanosine 5'-monophosphate; UMP, uridine 5'-monophosphate; UDP, uridine 5'-diphosphate; ADMA, NG, NG-dimethyl-L-arginine; CMP, cytidine 5'monophosphate. GMP $P^{(1)}$ and $G M P^{(2)}$ designate different molecules; their adducts are $(\mathrm{M}+\mathrm{H})^{+}$and $\left(\mathrm{M}+\mathrm{H}-\mathrm{H}_{2} \mathrm{O}\right)^{+}$, respectively. Differences with VIP $>1$ and $0.05<P<0.1$ are considered significant.

rectangle in the heat map represents one metabolite and is colored based on a normalized scale from -2 (low) to 2 (high). Differences between control and treated groups are the result of metabolite alterations after treatment with TTO. In positive mode (Figure 2A), seven metabolites [glycyl-Lleucine, L-carnosine, imidazoleacetic acid, L-methionine, N(omega)-hydroxyarginine, guanosine, and deoxyadenosine] were upregulated in the TTO-treated sample, accounting for $10.3 \%$ of metabolites in positive mode. Other metabolites such as Larginine, D-glucose 6-phosphate, phosphatidylcholine, alphalinolenic acid, D-mannitol, and L-glutamate were downregulated in the TTO-treated sample, accounting for $89.7 \%$ of metabolites in positive mode. In negative mode (Figure 2B), glycyl-L-leucine, 2 -oxoadipic acid, and guanosine were upregulated in the TTOtreated sample, accounting for $7.3 \%$ of metabolites in negative mode, while other metabolites such as isocitrate, L-malic acid, succinate, L-alanine, and trehalose were downregulated in the TTO-treated sample, accounting for $92.7 \%$ of metabolites in negative mode.

Isocitrate, L-malic acid, succinate, and $\alpha$-ketoglutarate are all involved in the TCA cycle. The contents of these compounds were all markedly decreased by $81.2,84.1,94.9$, and $91.9 \%$, respectively, in TTO-treated $B$. cinerea compared to the untreated control (Figure 2). Phosphatidylcholine and alphalinolenic acid are related to the fatty acid. The levels of phosphatidylcholine and alpha-linolenic acid compared with control downregulated $68.3 \%$ and $83.3 \%$ respectively in TTOtreated cells (Figure 3). As illustrated in Table 1, the detected L-carnosine and imidazoleacetic acid in TTO-treated groups compared with control was distinctly increased to 8.54- and 3.44-fold, respectively.

\section{Effects of TTO on Changes of Key Enzymes Activities Involved in TCA Cycle, $\mathrm{H}_{2} \mathrm{O}_{2}$ Accumulation, and Leakage of Cell Membrane}

As shown in Table 2, the activities of $\mathrm{MDH}, \mathrm{SDH}, \mathrm{CS}, \mathrm{ICDH}$ and $\alpha-\mathrm{KGDH}$ in untreated cells was $54.87 \mathrm{U} / \mathrm{g}, 23.75 \mathrm{U} / \mathrm{g}, 366.24$ $\mathrm{U} / \mathrm{g}, 647.50 \mathrm{U} / \mathrm{g}$ and $355.55 \mathrm{U} / \mathrm{g}$, respectively. After treatment with TTO at $5 \mathrm{~mL} / \mathrm{L}$ for $2 \mathrm{~h}$, the activities for these enzymes decreased about 79.3, 78.9, 83.3, 66.0, and 91.7\%, respectively. It indicated that all of these key enzymes involved in TCA cycle were significantly reduced in $B$. cinerea treated with TTO.

Meanwhile, $\mathrm{H}_{2} \mathrm{O}_{2}$ content increased from $43.65 \mathrm{mmol} / \mathrm{g}$ prot to $120.95 \mathrm{mmol} / \mathrm{g}$ prot because of TTO treatment (Table 2). Absorbance at $260 \mathrm{~nm}$ increased by $14 \%$ after TTO treatment for $2 \mathrm{~h}$, while the control group increased by only $1 \%$ (Figure 4 ). It was suggested that TTO led to the accumulation of $\mathrm{H}_{2} \mathrm{O}_{2}$ and the leakage of cell membrane.

\section{DISCUSSION}

Evidence suggests that essential oils damage fungal cell membranes, resulting in prolific leakage of ions, and nutrients. The leakage can be measured as an increase in absorbance at $260 \mathrm{~nm}$ and increasing conductivity in the medium (Hood et al., 2010; Tao et al., 2014). In our metabolomics analysis (Table 1), the majority of metabolites (83) were downregulated in the TTO-treated sample, perhaps due to the disruption of the cell membrane and metabolite leakage. But some metabolites (8) were upregulated in the TTO-treated sample. Shao et al. (2013a) showed that TTO treatment causes membrane leakage in $B$. cinerea, an increase in the activity of alkaline phosphatase, and an increase in palmitic acid (C16:0), stearic acid (C18:0), and oleic acid (C18:1) content was observed. Therefore, even after membrane integrity is compromised, some compounds may accumulate, possibly in response to stress.

The TCA cycle is the metabolic process by which ATP is generated in mitochondria, and is widely conserved in all oxidative organisms (Regev-Rudzki, 2005; Mailloux et al., 2007). The pathway contains several key enzymes (SDH, MDH, CS, $\mathrm{ICDH}, \alpha-\mathrm{KGDH})$ and produces critical intermediates such as isocitrate, succinate, malate, and alpha-ketoglutarate ( $\mathrm{Li}$ et al., 2015; Guo et al., 2016). As shown in Figure 2, TTO caused decreases in the levels of isocitrate, L-malic acid, succinate, and $\alpha$-ketoglutarate. Mousavi et al. demonstrated that the levels of metabolites related to the TCA cycle, such as malic acid and glucose 6-phosphate, decreased in E. coli after treatment with 

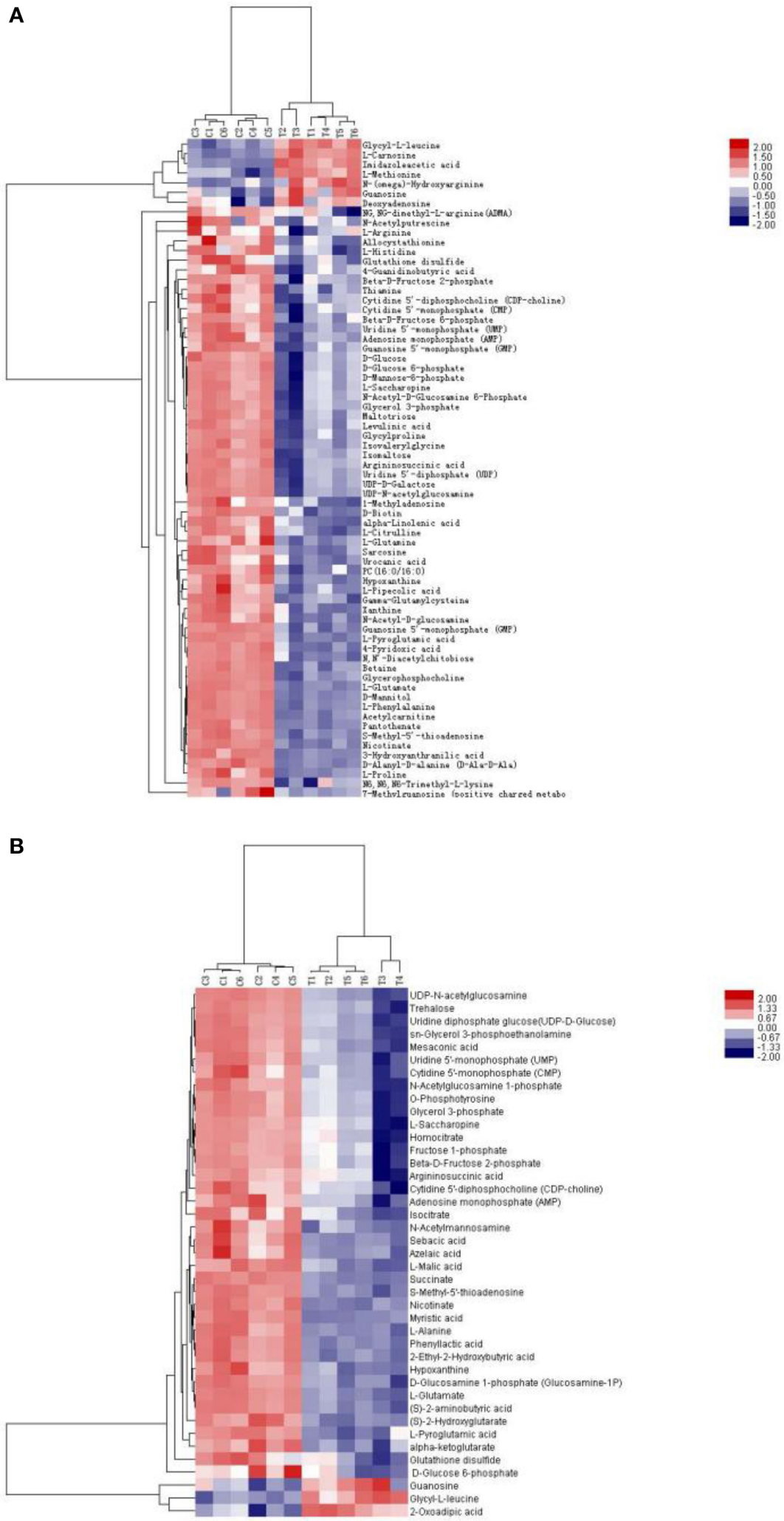

FIGURE 2 | Heat map visualizing changes in levels of potential biomarkers in positive mode (A) and negative mode (B). Rows: biomarkers; Columns: samples. The color key indicates the biomarker content ratio (blue: lowest; red: highest.) C, control sample; T, TTO-treated sample. 


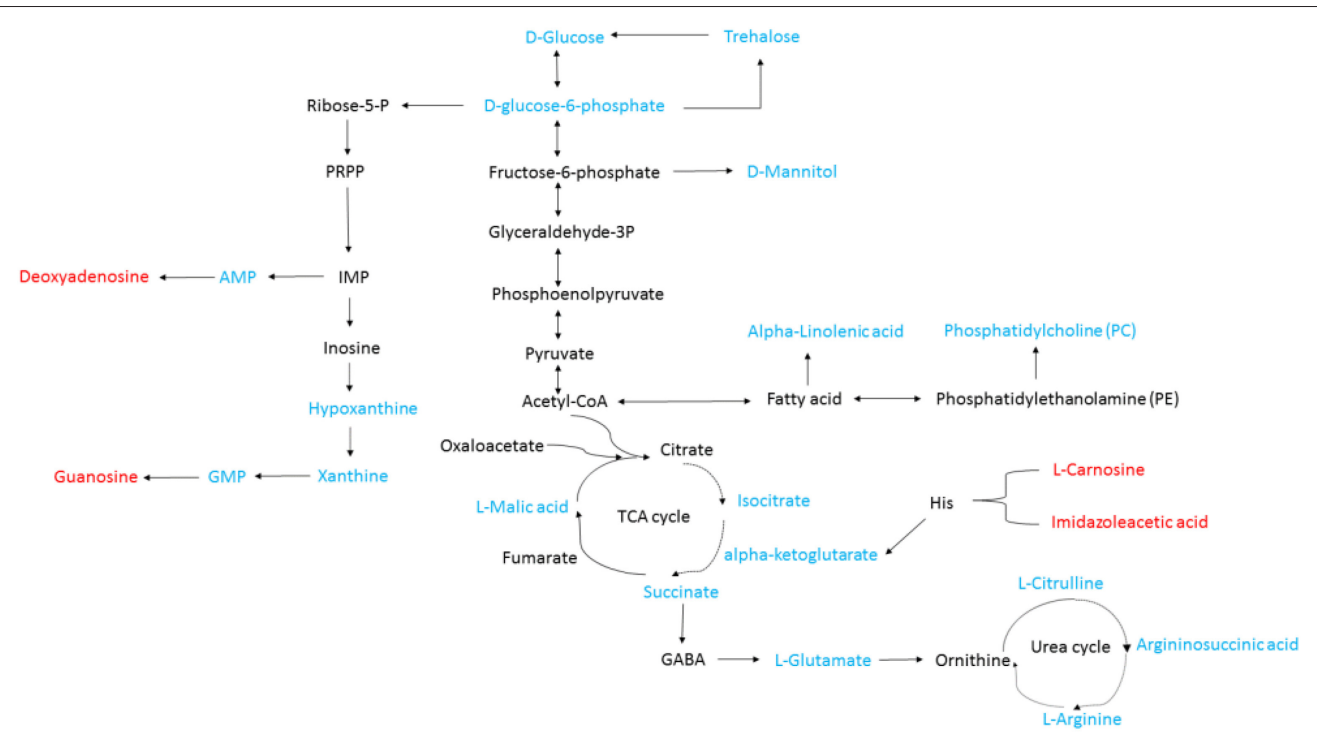

FIGURE 3 | General biosynthetic pathway for selected metabolites as obtained from the KEGG database. Metabolites in red denote metabolites that are relatively more abundant in the TTO-treated sample, while metabolites in blue denote metabolites that are relatively less abundant.

TABLE 2 | Effect of TTO on SDH, MDH, CS, ICDH, $\alpha$-KGDH, activities and $\mathrm{H}_{2} \mathrm{O}_{2}$ content in $B$. cinerea cells.

\begin{tabular}{|c|c|c|c|c|c|c|}
\hline Treatment & $\mathrm{SDH}(\mathrm{U} / \mathrm{g})^{*}$ & $\mathrm{MDH}(\mathrm{U} / \mathrm{g})^{*}$ & $\operatorname{CS}(U / g)^{*}$ & $\operatorname{ICDH}(\mathbf{U} / \mathbf{g})^{*}$ & $\alpha-\operatorname{KGDH}(\mathrm{U} / \mathrm{g})^{\star}$ & $\mathrm{H}_{2} \mathrm{O}_{2}$ (mmol/g prot)* \\
\hline тто & $5.00 \pm 1.53^{b}$ & $11.35 \pm 1.50^{b}$ & $61.16 \pm 9.22^{\mathrm{b}}$ & $220.29 \pm 41.46^{b}$ & $29.46 \pm 3.74^{\mathrm{b}}$ & $120.95 \pm 12.25^{a}$ \\
\hline
\end{tabular}

$a, b$ differences are significant at $P<0.05$ based on Duncan's multiple range tests.

*Values are presented as mean \pm standard deviation.

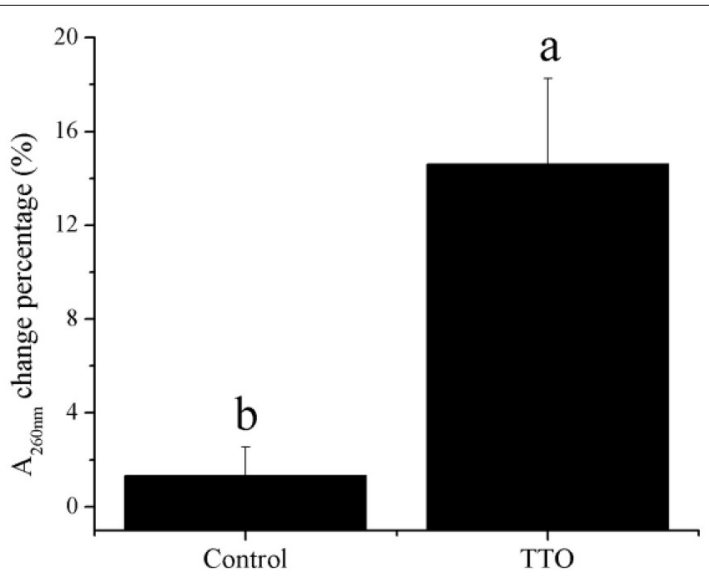

FIGURE 4 | Effects of TTO treatment on B. cinerea as measured by percentage absorbance change at $260 \mathrm{~nm}$. Vertical bars represent the standard deviation of the means. a,b: significant differences at $P<0.05$ level.

cinnamaldehyde, suggesting downregulation of the TCA cycle (Mousavi et al., 2016). It is therefore possible that the TCA cycle of $B$. cinerea is also inhibited by TTO treatment.

To test our hypothesis, we measured the activities of $\mathrm{MDH}$, $\mathrm{SDH}, \mathrm{CS}, \mathrm{ICDH}$, and $\alpha-\mathrm{KGDH}$, key enzymes associated with the TCA cycle. As shown in Table 2, treatment with TTO at $5 \mathrm{~mL} / \mathrm{L}$ for $2 \mathrm{~h}$ markedly decreased activities for these enzymes. In Penicillium digitatum cells, citral treatment inhibits the TCA pathway, as demonstrated by the reduction in citric acid content and reduction in the activities of $\mathrm{CS}, \mathrm{SDH}, \mathrm{ICDH}$, and $\alpha$ KGDH, although MDH activity increases (Zheng et al., 2015). However, MDH and SDH activities in Sclerotinia sclerotiorum and Rhizopus nigricans cells are decreased by dill seed essential oil and cinnamon oil, respectively (Li et al., 2014; Wang et al., 2015). Essential oils cause mitochondrial dysfunction by decreasing the activities of TCA-related enzymes, causing the accumulation of reactive oxygen species (ROS) and ultimately resulting in cell apoptosis (Tian et al., 2012; Zheng et al., 2015). Li et al. (2017) revealed that TTO severely damaged mitochondria of $B$. cinerea, resulting in matrix loss and increased mitochondrial irregularity. $\mathrm{H}_{2} \mathrm{O}_{2}$, an important ROS, can cause membrane lipid peroxidation and generation of protein carbonyl groups (Singh et al., 2015; Wang et al., 2016). TTO treatment increased $\mathrm{H}_{2} \mathrm{O}_{2}$ (Table 2), potentially resulting in oxidative stress and changes in the composition of cell membrane fatty acids. Together, these results suggest that TTO inhibits TCA cycle, possibly causing mitochondrial dysfunction and oxidation stress in B. cinerea.

Phosphatidylcholine is the major polar lipid in $B$. cinerea, while alpha-linolenate is the primary fatty acid (Griffiths et al., 2003). Both are major components of the cell membrane. 
Dill oil, which targets the plasma membrane of A. flavus, disrupts ergosterol biosynthesis (Khan et al., 2010; Tian et al., 2012). Citral exposure affects the expression of some genes involved in cell membrane-related pathways such as fatty acid biosynthesis and fatty acid metabolism, suggesting that it destroys cell membrane integrity (Ouyang et al., 2016). Citral, octanal, and $\alpha$-terpineol decrease the total lipid content in Geotrichum citri-aurantii cells, causing the destruction of the cell membrane (Zhou et al., 2014). We previously reported that TTO treatment decreases the unsaturated/saturated fatty acid ratio in $B$. cinerea, suggesting that cell membrane disruption is related to changes in fatty acid composition (Shao et al., 2013a). In this study, phosphatidylcholine and alpha-linolenic acid were downregulated in TTO-treated cells (Figure 3), as well as the leakage of cell membrane (Figure 4). These results imply that TTO promotes membrane leakage either directly, or indirectly by interfering with membrane lipid biosynthesis and fatty acid composition.

A dramatic increase in $\mathrm{H}_{2} \mathrm{O}_{2}$ content was detected in $B$. cinerea cells incubated with TTO (Table 2), which demonstrates that TTO treatment increases the level of free radicals, resulting in oxidative stress. L-carnosine (beta-alanylL-histidine) is a natural dipeptide with antioxidant activity and functions as a scavenger for free-radicals (Hipkiss, 2009; Boldyrev et al., 2013). L-carnosine levels increased in the TTO-treated sample, perhaps as a protective response against $\mathrm{H}_{2} \mathrm{O}_{2}$-induced oxidative stress. L-carnosine has a similar function in red blood cells subjected to oxidative stress by $\mathrm{H}_{2} \mathrm{O}_{2}$ (Aydogan et al., 2008).

The binding of $\gamma$-aminobutyric acid (GABA) to the GABA receptor triggers changes in plasma membrane ionic permeability (Perfilova and Tiurenkov, 2011). At least three types of GABA receptors, designated $\mathrm{GABA}_{A}, \mathrm{GABA}_{B}$, and $\mathrm{GABA}_{C}$, have been

\section{REFERENCES}

Adebayo, O., Bélanger, A., and Khanizadeh, S. (2013). Variable inhibitory activities of essential oils of three Monarda species on the growth of Botrytis cinerea. Can. J. Plant Sci. 93, 987-995. doi: 10.4141/cjps2013-044

Aguilar-González, A. E., Palou, E., and López-Malo, A. (2015). Antifungal activity of essential oils of clove (Syzygium aromaticum) and/or mustard (Brassica nigra) in vapor phase against gray mold (Botrytis cinerea) in strawberries. Innov. Food Sci. Emerg. 32, 181-185. doi: 10.1016/j.ifset.2015.09.003

Aydogan, S., Yapislar, H., Artis, S., and Aydogan, B. (2008). Impaired erythrocytes deformability in $\mathrm{H}_{(2)} \mathrm{O}_{(2)}$-induced oxidative stress: protective effect of Lcarnosine. Clin. Hemorheol. Microcirc. 39, 93-98. doi: 10.3233/CH-2008-1072

Bishop, C. D., and Reagan, J. (1998). Control of the storage pathogen Botrytis cinerea on Dutch white cabbage (Brassica oleracea var. capitata) by the essential oil of Melaleuca alternifolia. J. Essent. Oil Res. 10, 57-60. doi: 10.1080/10412905.1998.9700838

Bo, T., Liu, M., Zhong, C., Zhang, Q., Su, Q.-Z., Tan, Z.-L., et al. (2014). Metabolomic analysis of antimicrobial mechanisms of $\varepsilon$-Poly-1lysine on Saccharomyces cerevisiae. J. Agric. Food Chem. 62, 4454-4465. doi: 10.1021/jf500505n

Boldyrev, A. A., Aldini, G., and Derave, W. (2013). Physiology and pathophysiology of carnosine. Physiol. Rev. 93, 1803-1845. doi: 10.1152/physrev.00039.2012

Bouchra, C., Achouri, M., Idrissi Hassani, L. M., and Hmamouchi, M. (2003). Chemical composition and antifungal activity of essential oils of seven characterized (Qian and Dowling, 1993). Imidazoleacetic acid is a major catabolite of histamine and functions as a $\mathrm{GABA}_{C}$ receptor antagonist (Liu et al., 2006). Our data show that TTO treatment increases membrane permeability in $B$. cinerea (Figure 4) and also increases the level of imidazoleacetic acid in $B$. cinerea (Figure 3). The increase in the level of imidazoleacetic acid may inhibit binding to the $\mathrm{GABA}_{\mathrm{C}}$ receptor, conferring partial protection against membrane leakage induced by TTOtreatment.

\section{CONCLUSION}

This report presents the first metabolomics analysis of $B$. cinerea after treatment by TTO, and demonstrates that TTO disrupts the TCA cycle, affects the level of various cellular components, and causes cell membrane leakage. The damage results in mitochondrial dysfunction and oxidative stress. L-carnosine and imidazoleacetic acid levels increase in response to these events.

\section{AUTHOR CONTRIBUTIONS}

JX and XS designed the experiments. JX, YL, and YW performed the experiments. FX and HW analyzed the data. JX, XS, and HW drafted the manuscript. All authors read and approved the final manuscript.

\section{ACKNOWLEDGMENTS}

This study was funded by the National Science Foundation of China (No. 31371860), the Public Welfare Applied Research Project of Zhejiang Province, China (No. 2017C32010) and the K. C. Wong Magna Fund in Ningbo University.

Moroccan Labiatae against Botrytis cinerea Pers: Fr. J. Ethnopharmacol. 89, 165-169. doi: 10.1016/S0378-8741(03)00275-7

Cao, Y., Zhu, Z., Chen, X., Yao, X., Zhao, L., Wang, H., et al. (2013). Effect of amphotericin B on the metabolic profiles of Candida albicans. J. Proteome Res. 12, 2921-2932. doi: 10.1021/pr4002178

Cheng, S., and Shao, X. (2011). "In vivo antifungal activities of the tea tree oil vapor against Botrytis cinerea," in International Conference on New Technology of Agricultural Engineering (Zibo), 949-951.

Fiehn, O., Kopka, J., Dörmann, P., Altmann, T., Trethewey, R. N., and Willmitzer, L. (2000). Metabolite profiling for plant functional genomics. Nat. Biotechnol. 18, 1157-1161. doi: 10.1038/81137

Griffiths, R. G., Dancer, J., O’Neill, E., and Harwood, J. L. (2003). Lipid composition of Botrytis cinerea and inhibition of its radiolabelling by the fungicide iprodione. New Phytol. 160, 199-207. doi: 10.1046/j.1469-8137.2003. 00848.x

Guo, H., Madzak, C., Du, G., and Zhou, J. (2016). Mutagenesis of conserved active site residues of dihydrolipoamide succinyltransferase enhances the accumulation of $\alpha$-ketoglutarate in Yarrowia lipolytica. Appl. Microbiol. Biotechnol. 100, 649-659. doi: 10.1007/s00253-015-6995-1

Gustafson, J., Liew, Y., Chew, S., Markham, J., Bell, H., Wyllie, S., et al. (1998). Effects of tea tree oil on Escherichia coli. Lett. Appl. Microbiol. 26, 194-198. doi: 10.1046/j.1472-765X.1998.00317.x

Hammer, K., Carson, C., and Riley, T. (2003). Antifungal activity of the components of Melaleuca alternifolia (tea tree) oil. J. Appl. Microbiol. 95, 853-860. doi: 10.1046/j.1365-2672.2003.02059.x 
Hipkiss, A. R. (2009). Chapter 3 carnosine and its possible roles in nutrition and health. Adv. Food Nutr. Res. 57, 87-154. doi: 10.1016/S1043-4526(09)57003-9

Hood, J. R., Burton, D. M., Wilkinson, J. M., and Cavanagh, H. M. (2010). The effect of Leptospermum petersonii essential oil on Candida albicans and Aspergillus fumigatus. Med. Mycol. 48, 922-931. doi: 10.3109/13693781003774697

Khan, A., Ahmad, A., Akhtar, F., Yousuf, S., Xess, I., Khan, L. A., et al. (2010). Ocimum sanctum essential oil and its active principles exert their antifungal activity by disrupting ergosterol biosynthesis and membrane integrity. Res. Microbiol. 161, 816-823. doi: 10.1016/j.resmic.2010.09.008

Li, L., Xing, W. W., Shao, Q. S., Shu, S., Sun, J., and Guo, S. R. (2015). The effects of grafting on glycolysis and the tricarboxylic acid cycle in $\mathrm{Ca}(\mathrm{NO} 3)_{2}$-stressed cucumber seedlings with pumpkin as rootstock. Acta Physiol. Plant. 37, 1-10. doi: 10.1007/s11738-015-1978-5

Li, W. R., Shi, Q. S., Dai, H. Q., Liang, Q., Xie, X. B., Huang, X. M., et al. (2016). Antifungal activity, kinetics and molecular mechanism of action of garlic oil against Candida albicans. Sci. Rep. 6:22805. doi: 10.1038/srep22805

Li, Y., Nie, Y., Zhou, L., Li, S., Tang, X., Ding, Y., et al. (2014). The possible mechanism of antifungal activity of cinnamon oil against Rhizopus nigricans. J. Chem. Pharm. Res. 6, 12-20.

Li, Y., Shao, X., Xu, J., Wei, Y., Xu, F., and Wang, H. (2017). Tea tree oil exhibits antifungal activity against Botrytis cinerea by affecting mitochondria. Food Chem. 234, 62-67. doi: 10.1016/j.foodchem.2017.04.172

Liu, H., Du, Y., Wang, X., and Sun, L. (2004). Chitosan kills bacteria through cell membrane damage. Int. J. Food Microbiol. 95, 147-155. doi: 10.1016/j.ijfoodmicro.2004.01.022

Liu, J., Li, G. L., and Yang, X. L. (2006). An ionotropic GABA receptor with novel pharmacology at bullfrog cone photoreceptor terminals. Neurosignals 15 , 13-25. doi: 10.1159/000094384

Liu, S., Shao, X., Wei, Y., Li, Y., Xu, F., and Wang, H. (2016). Solidago canadensis L. essential oil vapor effectively inhibits Botrytis cinerea growth and preserves postharvest quality of strawberry as a food model system. Front Microbiol. 7:1179. doi: 10.3389/fmicb.2016.01179

Mailloux, R. J., Bériault, R., Lemire, J., Singh, R., Chénier, D. R., Hamel, R. D., et al. (2007). The tricarboxylic acid cycle, an ancient metabolic network with a novel twist. PLOS ONE 2:e690. doi: 10.1371/journal.pone.0000690

Mousavi, F., Bojko, B., Bessonneau, V., and Pawliszyn, J. (2016). Cinnamaldehyde characterization as an antibacterial agent toward E. coli metabolic profile using 96-blade solid-phase microextraction coupled to liquid chromatography-mass spectrometry. J. Proteome Res. 15, 963-975. doi: 10.1021/acs.jproteome.5b00992

Ouyang, Q., Tao, N., and Jing, G. (2016). Transcriptional profiling analysis of Penicillium digitatum, the causal agent of citrus green mold, unravels an inhibited ergosterol biosynthesis pathway in response to citral. BMC Genomics 17:599. doi: 10.1186/s12864-016-2943-4

Pazyar, N., Yaghoobi, R., Bagherani, N., and Kazerouni, A. (2013). A review of applications of tea tree oil in dermatology. Int. J. Dermatol. 52, 784-790. doi: 10.1111/j.1365-4632.2012.05654.x

Perfilova, V. N., and Tiurenkov, I. N. (2011). [GABAC receptors: structure and functions]. Eksp. Klin. Farmakol. 74, 45-49.

Qian, H., and Dowling, J. E. (1993). Novel GABA responses from rod-driven retinal horizontal cells. Nature 361, 162-164. doi: 10.1038/361162a0

Regev-Rudzki, N. (2005). Yeast aconitase in two locations and two metabolic pathways: seeing small amounts is believing. Mol. Biol. Cell 16, 4163-4171. doi: 10.1091/mbc.E04-11-1028

Saito, S., Margosan, D., Michailides, T. J., and Xiao, C. L. (2016). Botrytis californica, a new cryptic species in the B. cinerea species complex causing gray mold in blueberries and table grapes in California. Mycologia 108, 330-343. doi: $10.3852 / 15-165$
Schneider, K., Krömer, J. O., Wittmann, C., Alves-Rodrigues, I., Meyerhans, A., Diez, J., et al. (2009). Metabolite profiling studies in Saccharomyces cerevisiae: an assisting tool to prioritize host targets for antiviral drug screening. Microb. Cell Fact. 8:12. doi: 10.1186/1475-2859-8-12

Shao, X., Cheng, S., Wang, H., Yu, D., and Mungai, C. (2013a). The possible mechanism of antifungal action of tea tree oil on Botrytis cinerea. J. Appl. Microbiol. 114, 1642-1649. doi: 10.1111/jam.12193

Shao, X., Wang, H., Xu, F., and Cheng, S. (2013b). Effects and possible mechanisms of tea tree oil vapor treatment on the main disease in postharvest strawberry fruit. Postharvest. Biol. Technol. 77, 94-101. doi: 10.1016/j.postharvbio.2012.11.010

Shi, C., Zhao, X., Yan, H., Meng, R., Zhang, Y., Li, W., et al. (2016). Effect of tea tree oil on Staphylococcus aureus growth and enterotoxin production. Food Control 62, 257-263. doi: 10.1016/j.foodcont.2015.10.049

Singh, M. P. V. V. B., Prasad, S. M., Singh, V. P., and Singh, M. (2015). Antioxidant system against active oxygen species in Cyanobacterium Aphanothece stagnina: response to excess light under cadmium stress. Proc. Natl. Acad. Sci. U.S.A.. 85, 535-543. doi: 10.1007/s40011-014-0367-y

Soylu, E. M., Kurt, S., and Soylu, S. (2010). In vitro and in vivo antifungal activities of the essential oils of various plants against tomato grey mould disease agent Botrytis cinerea. Int. J. Food Microbiol. 143, 183-189. doi: 10.1016/j.ijfoodmicro.2010.08.015

Szczerbanik, M., Jobling, J., Morris, S., and Holford, P. (2007). Essential oil vapours control some common postharvest fungal pathogens. Aust. J. Exp. Agric. 47, 103-109. doi: 10.1071/EA05236

Tao, N., OuYang, Q., and Jia, L. (2014). Citral inhibits mycelial growth of Penicillium italicum by a membrane damage mechanism. Food Control 41, 116-121. doi: 10.1016/j.foodcont.2014.01.010

Tian, J., Ban, X., Zeng, H., He, J., Chen, Y., and Wang, Y. (2012). The mechanism of antifungal action of essential oil from dill (Anethum graveolens L.) on Aspergillus flavus. PLoS ONE 7:e30147. doi: 10.1371/journal.pone.0030147

Wang, Z., Li, S., Yu, C., Tian, X., Rong, Z., Liao, D. F., et al. (2016). Oxidative stress and carbonyl lesions in ulcerative colitis and associated colorectal cancer. Oxid. Med. Cell. Longev. 2016, 1-15. doi: 10.1155/2016/9875298

Wang, Z., Ma, B., Ban, X., Huang, B., He, J., Tian, J., et al. (2015). Interference and mechanism of dill seed essential oil and contribution of carvone and limonene in preventing sclerotinia rot of rapeseed. PLOS ONE 10:e0131733. doi: 10.1371/journal.pone. 0131733

Yu, D., Wang, J., Shao, X., Xu, F., and Wang, H. (2015). Antifungal modes of action of tea tree oil and its two characteristic components against Botrytis cinerea. J. Appl. Microbiol. 119, 1253-1262. doi: 10.1111/jam.12939

Zheng, S., Jing, G., Wang, X., Ouyang, Q., Jia, L., and Tao, N. (2015). Citral exerts its antifungal activity against Penicillium digitatum by affecting the mitochondrial morphology and function. Food Chem. 178, 76-81. doi: 10.1016/j.foodchem.2015.01.077

Zhou, H., Tao, N., and Jia, L. (2014). Antifungal activity of citral, octanal and $\alpha$-terpineol against Geotrichum citri-aurantii. Food Control 37, 277-283. doi: 10.1016/j.foodcont.2013.09.057

Conflict of Interest Statement: The authors declare that the research was conducted in the absence of any commercial or financial relationships that could be construed as a potential conflict of interest.

Copyright ( $\odot 2017 \mathrm{Xu}$, Shao, Li, Wei, Xu and Wang. This is an open-access article distributed under the terms of the Creative Commons Attribution License (CC BY). The use, distribution or reproduction in other forums is permitted, provided the original author(s) or licensor are credited and that the original publication in this journal is cited, in accordance with accepted academic practice. No use, distribution or reproduction is permitted which does not comply with these terms. 